\title{
NUMB is a break of WNT - Notch signaling cycle
}

\author{
MASUKO KATOH $^{1}$ and MASARU KATOH ${ }^{2}$ \\ ${ }^{1}$ M\&M Medical BioInformatics, Hongo 113-0033; ${ }^{2}$ Genetics and Cell Biology Section, \\ National Cancer Center Research Institute, Tokyo 104-0045, Japan
}

Received April 19, 2006; Accepted May 25, 2006

\begin{abstract}
Notch, FGF and WNT signaling pathways cross-talk during embryogenesis, tissue regeneration and carcinogenesis. Notch-ligand binding to Notch receptors leads to the cleavage of Notch receptors and the following nuclear translocation of Notch intracellular domain (NICD) to induce transcriptional activation of Notch target genes. Notch signaling inhibitors, NUMB and NUMB-like (NUMBL), are docking proteins with PTB domain. We searched for the TCF/LEF-binding site within the promoter region of $N U M B$ and $N U M B L$ genes. Because two TCF/LEF-binding sites were identified within human $N U M B$ promoter based on bioinformatics and human intelligence (Humint), comparative integromics analyses on $N U M B$ orthologs were further performed. Chimpanzee NUBM gene, consisting of 13 exons, was identified within NW_115880.1 genome sequence. XM_510045.1 was not the correct coding sequence for chimpanzee NUMB. Chimpanzee NUMB gene was found to encode a 651-amino-acid protein showing $99.5,93.9$ and $82.6 \%$ total-amino-acid identity with human NUMB, mouse Numb and chicken numb, respectively. Human $N U M B$ mRNA was expressed in placenta, ES cells, neural tissues, trachea, testis, uterus, thymus, coronary artery as well as in a variety of tumors, such as cervical cancer, tong tumor, brain tumor, colorectal and breast cancer. Although distal TCF/LEF-binding site within human $N U M B$ promoter was conserved only among primate $N U M B$ orthologs, proximal TCF/LEF-binding site was conserved among primate and rodent $N U M B$ orthologs. NUMB, JAG1, FGF18, FGF20 and SPRY4 are potent targets of the canonical WNT signaling pathway in progenitor cells. NUMB inhibits Notch signaling in progenitor cells to induce differentiation, while JAG1 activates Notch signaling in stem cells to maintain self-renewal potential. Because Notch signaling inhibitor NUMB was identified as the safe apparatus for the WNT - Notch signaling cycle,
\end{abstract}

Correspondence to: Dr Masaru Katoh, Genetics and Cell Biology Section, National Cancer Center Research Institute, 5-1-1 Tsukiji, Chuo-ku, Tokyo 104-0045, Japan

E-mail:mkatoh@ncc.go.jp

Key words: bioinformatics, comparative genomics, comparative proteomics, WNT, Notch, integrome network epigenetic silencing, deletion and loss-of-function mutation of NUMB gene could lead to carcinogenesis through the dysregulation of the WNT - Notch signaling cycle.

\section{Introduction}

Notch signaling pathway is implicated in the maintenance of self-renewal potential in stem cells, binary cell-fate determination in progenitor cells, and induction of terminal differentiation in proliferating cells (1-7). Notch-ligand binding to Notch receptors leads to the cleavage of Notch receptors by metalloprotease and $\gamma$-secretase to induce nuclear translocation of Notch intracellular domain (NICD). Nuclear complex, consisting of CSL (RBPSUH), NICD, Mastermind (MAML), p300 and histone acetyltransferase (HAT), then induces transcriptional activation of Notch target genes, such as HES1, HES5, HES7, HEY1, HEY2 and HEYL. HES/HEY family members are bHLH-type transcriptional repressors for tissue-specific transcription factors. Therefore, Notch signaling activation in stem cells leads to the maintenance of self-renewal potential.

Notch ligand JAG1 as well as FGF20 and FGF18 are evolutionarily conserved targets of the canonical WNT signaling pathway (7-9). Notch4, Wnt1, Wnt3, Wnt10b, Fgf3, $F g f 4$ and $F g f 8$ are up-regulated during mouse mammary carcinogenesis due to MMTV integration $(10,11)$. Notch, FGF and WNT signaling pathways cross-talk during embryogenesis, tissue regeneration and carcinogenesis (1-31).

NUMB and NUMB-like (NUMBL), consisting of phosphotyrosine-binding (PTB) domain and SH3-binding proline-rich region, are docking proteins functioning as Notch signaling inhibitors $(32,33)$. Here, we searched for the TCF/LEF-binding site within $N U M B$ and $N U M B L$ promoters. Because two TCF/LEF-binding sites were identified within human $N U M B$ promoter, comparative integromics analyses on $N U M B$ orthologs were further performed.

\section{Materials and methods}

WNT target gene screening. Genome sequences corresponding to human $N U M B$ and $N U M B L$ genes were searched for with BLAST programs (http://www.ncbi.nlm.nih.gov) as described previously (34-36). TCF/LEF-binding sites within the 5'flanking promoter region of above genes were then searched for based on bioinformatics and human intelligence as described previously (37-39). 
A

\begin{tabular}{|c|c|c|c|}
\hline $\begin{array}{l}\text { Exon } \\
\text { No }\end{array}$ & & $\begin{array}{l}\text { leotide sequence of chimpan } \\
\text { toround exon-intron bound }\end{array}$ & ees \\
\hline $\begin{array}{r}1 \\
2 \\
3 \\
4 \\
5 \\
6 \\
7 \\
8 \\
9 \\
10 \\
11 \\
12 \\
13\end{array}$ & 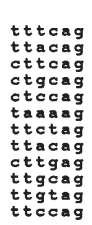 & 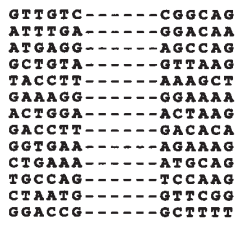 & 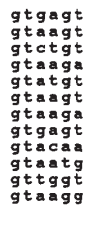 \\
\hline
\end{tabular}

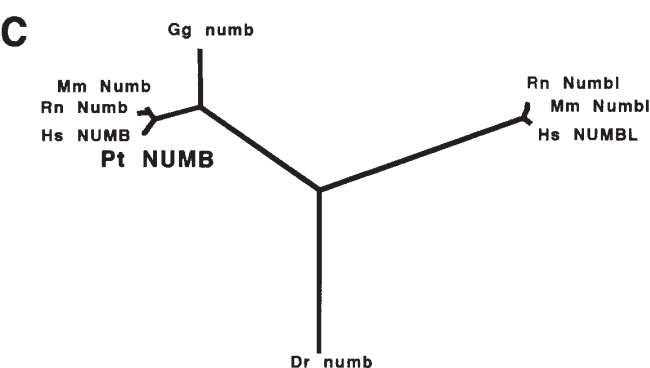

$\mathbf{B}$

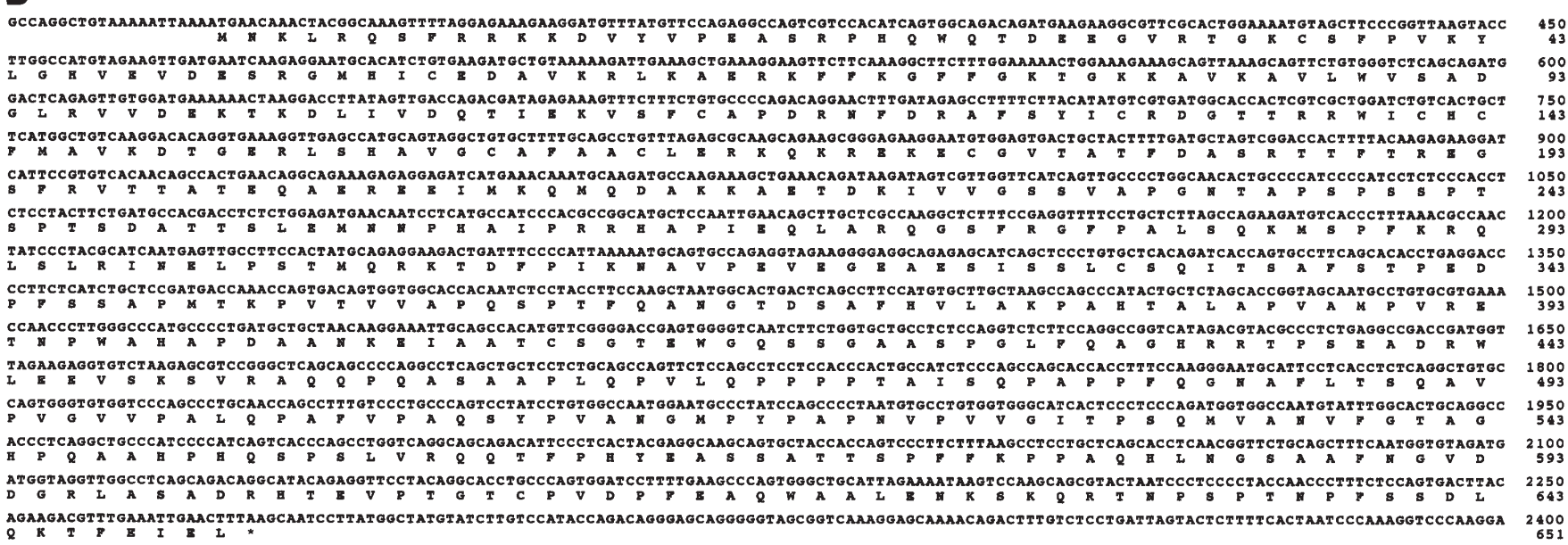

Figure 1. Chimpanzee $N U M B$ gene. (A), Exon-intron structure of chimpanzee $N U M B$ gene. Nucleotide sequences around exon-intron boundaries are shown by upper-case letters (exon) and by lower-case letters (intron). (B), Nucleotide and amino-acid sequences of chimpanzee NUMB complete CDS. Nucleotides and amino-acid residues are numbered on the right. Nucleotide position 301-2400 of chimpanzee NUMB complete CDS is shown. (C), Phylogenetic tree of vertebrate NUMB homologs.

Identification of chimpanzee NUMB ortholog. Chimpanzee genome sequence homologous to human $N U M B$ was searched for with the BLAST programs as described previously (40-42). Exon-intron boundaries were determined by examining the consensus sequence of exon-intron junctions ('gt ... ag' rule of intronic sequence) and the codon usage within the coding region as described previously (43-45). Coding sequence of chimpanzee NUMB was determined by assembling exonic regions.

Comparative proteomics analysis. Phylogenetic analyses on vertebrate NUMB and NUMBL orthologs were performed by using the CLUSTALW program.

Comparative genomics analyses. Promoter region of human, chimpanzee, mouse and rat $N U M B$ orthologs were aligned by using the Genetyx program and manual curation. TCF/LEFbinding sites within the promoter region were determined as mentioned above.

In silico expression analysis. Expressed sequence tags (ESTs) derived from human $N U M B$ gene were searched for by using the BLAST programs. The sources of NUMB ESTs were listed up for in silico expression analysis on NUMB mRNA.

\section{Results}

Screening of TCF/LEF-binding site within promoter region of NUMB and NUMBL genes. Human NUMB RefSeq
(NM_001005743.1) and NUMBL RefSeq (NM_004756.3) were used as query sequences for the BLAST programs to identify genome clones corresponding to $N U M B$ and $N U M B L$ genes. The 5'-flanking promoter region of human $N U M B$ and NUMBL genes were identified within AC005280.3 and AC010412.9 genome sequences, respectively. TCF/LEFbinding sites within the 5'-promoter region of human $N U M B$ and NUMBL genes were then searched for. Double TCF/LEFbinding sites were identified within human $N U M B$ promoter based on bioinformatics and human intelligence.

Identification of chimpanzee NUMB gene. BLAST programs using human NUMB RefSeq revealed that chimpanzee $N U M B$ gene was located within NW_115880.1 genome sequence. Exon-intron boundaries of chimpanzee $N U M B$ gene were determined based on the consensus sequence of exon-intron junctions. Chimpanzee $N U M B$ gene was found consisting of 13 exons (Fig. 1A).

Chimpanzee NUMB predicted sequence XM_510045.1 corresponded to intron 1, exon 2, exons 4-9 and intron 9 of chimpanzee NUMB gene. Because XM_510045.1 was not the correct chimpanzee NUMB sequence, complete coding sequence (CDS) of chimpanzee NUMB was determined in this study by assembling nucleotide sequences of 13 exons (Fig. 1B).

Genetyx program revealed that nucleotide position 3212276 was the coding region of chimpanzee NUMB complete CDS (Fig. 1B). Chimpanzee $N U M B$ gene was found to encode a 651-amino-acid protein. 


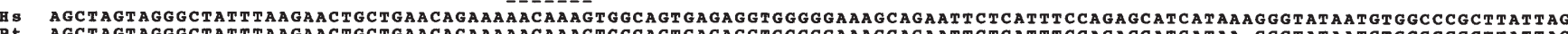
Pt AGCTAGTAGGGCTATT TAAGAACTGCTGAACAGAAAAACAAAGTGGAGTGAGAGGTGGGGGAAGCAGAATTCTCATTTCCAGAGCATCATAA-GGGTATAATGTGGCCCGCTTATTAG

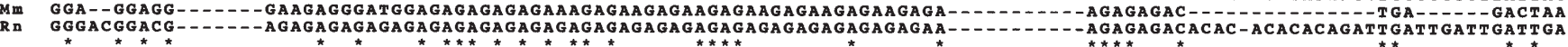
HA AAGTGCTGGCCGGGCGCGTGGCTTACGCCTGTAATACCAGCACTTTGGGAGGCCGAGGCGGGGGATCACCTGAGGTTGGGATTCGAGACTAGCCTGACCAACATGGAGAACCCC
Pt AAATTGCTGGCCGGCGCGGTGGTTACGCCTGTAATACCAGCACTTGGGAGGCCGAGGCGGCGGATCACCTGAGGTGGGATTCGAGACTAGCCTGACCAACATGGAGAACTCC Mm TTGGTATCTGCC-AGATA-- -

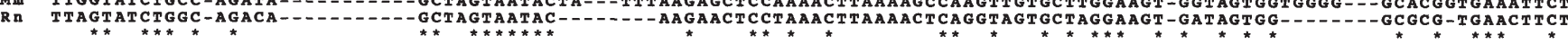
H GTCTCATGCCTATAATCCCAGCTACTCGGGAGCTAAGGCAGAAGATCGCTTGAACTTGGAGGCGTAGGTTGCGATGAGCCGAGATAGCACCATTGCACTCCAGCCTGGGCAACAAGA Pt GTCTCATGCCTATAATCCCAGCTACTCGGGAGGCTAAGGCAGGAGATCGCTTGAACCTGGGAGGCGTAGGTTGCGATGAGCCGAGATAGCACCATTGCACTCCAGCCTGGGCAAC--GA Rn

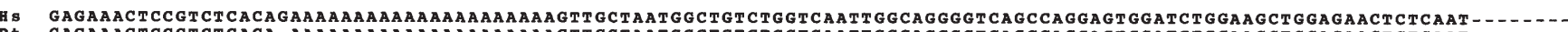
Pt GAGAACTCCGTCTCACA-AAAAAAAAAAAAAAAAAAAGTTGCTAATGCTGTCTGGTCAATTGGAGGGTCAGCCAGGAGTGATCTGGAAGTGGAGAACTCTCAAT--1-2.-

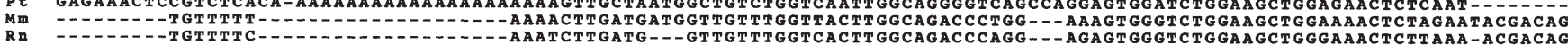
ACGCCACTTCAG--- TTTATTGACGACATTTATAATTGCAACCGAAGTT-GACTGATTTCTGCTAAATGAAAT-TTGCGCTTTGCCCTTTAGCATTGAGG-CTCCTGGAAGAACT ACGCCACTTCAG----TTTATTGACGACATTTATAATTGCAACCGAAGTT-GACTGATTTCTGCTAAATGAAAGT-TTGCGCTTTGCGCTTTAGCATTGAGG-CTCCTGGAAAGAAC ACACAATTATAAATATATTTCTTGACGATATTTATAAATGCAATGGAAAC- ${ }_{*}$

M TCAATGAAATTGGTGCATTGAGACCAAAGAAAGAAGGGACATTTTTATCTCTCTGATACCTTTCTCCCCAGGGCTTGGAAACACTTCTTAAACACAACCTCTAATGTTGGTGTTTT

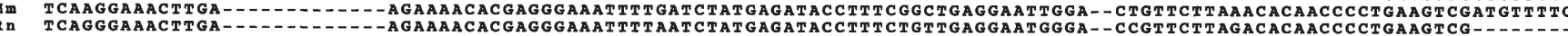

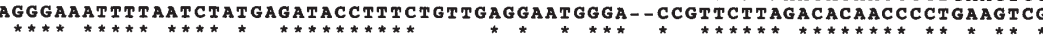

18 TAAGTACTACCCCTATGAATAAAGCTAGCAGAGATAAGCTTCTCCTTGCAGGAAGTGAGCTGGAGAAGTGGAAAAGTTTACAGAGAAAACTCTACAACAAAGACTTAACCCTTCCCGGG TEAGTACTACCCCTATGAATAAAGCTAGCAGAGATAAGCTTCTCCTTGCAGGAAGTGAGCTGGAGAAGTGGAGGGTTACAGAGAAAACTCTACAACAAAGAGTTAACCCTTCCCGTGI

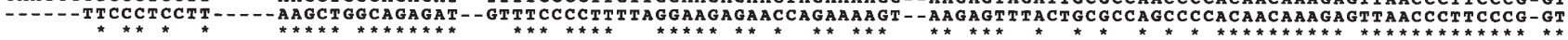
AACCACGAATGTGAGCGTCTGCCCCTCCTTTGCTTTCTACTGCGCAACCAAACTGTGCACAAG-CGTGATCAACCTTAACCGTCACGTCTCTTGCCAGTPAGCTTAACAGACACTTTTA AACCTCGGACGT

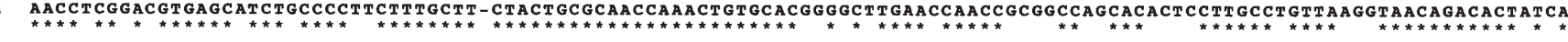
GCAGCCTATCATAACTTCGAAT TGCCCAACCCTCAACCAAT TACAACTAAAGCTTGGGCTAGGACGCTCCAGGTCCGGGCGGGGGCGAATACCGGGTTTAGGAAAAGGGCGGTAAAGA-

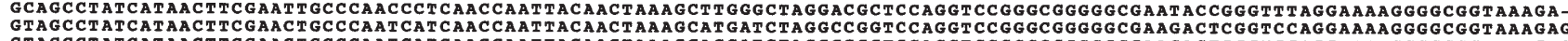
GTAGCCTATCATAACTTCGAACTGCCCAATCATCAACCAATTACAACTAAAGCACGATCTAGGCCGGTCCAGGTCCGGGCGGGGGCGAAGACTCGGTCCAGGAAAAGGGCGGTAAAGAC

H GCGATGACGGGCGGGTATACTAAACCAATCAGAGAGGAGAGCTGCCGCCCCACTATCCAATGGATAGTTAGAAGTATGAAGAGGGTGGCCTGTTGTCATGGGGGAGGTGGTGGCGCT GCGATGACGGGCGGGTATACTAAACCAATCAGAGAGGAGAGCTGCCGCCCCACTATCCAATGGATAGGT TAGAAGTATGAAAGAGGGT GGGCCTGTTGTCATGGGGGAGGTGGT GGCGCT AGGAT GACGGACAGGAAGAGTCAACCAAT CGCAGCAGAGGGCCGCCGCCC TCCACCCAATGGATAAGT TGGAAGTGTGGTATGGGGCGGCCTGTTGTCATGGGGGAGGTGGTGGCGCT GGATGACAGGCAGGAAGAGTCAACCAATCATAGCAGAGAGCCGCCGCCCCTCCAGCCAATGGATAAGTTGAAGTTGGGATGGGCGGGCCTGTTGTCATGGGGAGGTGGTGCGCT

Figure 2. Alignment of mammalian $N U M B$ promoters. Hs, human; Pt, chimpanzee; Mm, mouse; Rn, rat. Region corresponding to exon 1 of human $N U M B$ gene is boxed. Two TCF/LEF-binding sites within human $N U M B$ promoter are shown by double over-lines. Distal TCF/LEF-binding site within human $N U M B$ promoter is conserved only among primate $N U M B$ orthologs, while proximal TCF/LEF-binding site is conserved among primate and rodent $N U M B$ orthologs

Comparative integromics analysis on $N U M B$ and NUMBL homologs. Intra-species comparative genomics revealed that LTBP4-NUMBL-ADCK4 locus at human chromosome $19 \mathrm{q} 13.2$ and NUMB-LTBP2-ADCK1 locus at human chromosome $14 \mathrm{q} 24$ were paralogous regions with recombinations within the human genome.

Phylogenetic analysis revealed that zebrafish numb was significantly divergent from mammalian NUMB and NUMBL orthologs (Fig. 1C).

Expression of human NUMB mRNA. In silico expression analysis revealed that human $N U M B$ mRNA was expressed in placenta, ES cells, neural tissues, trachea, testis, uterus, thymus, coronary artery as well as in a variety of tumors, such as cervical cancer, tong tumor, brain tumor, colorectal cancer and breast cancer.

Comparative genomics analyses on NUMB promoters. Human $N U M B$ promoter and chimpanzee NUMB promoter were located within AC005280.3 and NW_115880.1 genome sequences, respectively, as mentioned above. BLAST programs using mouse Numb cDNA (BC033459.1) and rat Numb RefSeq (NM_133287.1) revealed that mouse Numb promoter and rat Numb promoter were located within AC133183.3 and NW_047762.2 genome sequences, respectively. GC content of human, chimpanzee, mouse and rat $N U M B$ promoters were $51.3,51.1,53.2$ and $52.7 \%$, respectively.
Double TCF/LEF-binding sites within human $N U M B$ promoter were located about 1100 and $350 \mathrm{bp}$ upstream of the transcription start site (Fig. 2). Proximal TCF/LEF-binding site was conserved among primate and rodent $N U M B$ orthologs, while distal TCF/LEF-binding site was conserved only among primate $N U M B$ orthologs (Fig. 2).

\section{Discussion}

TCF/LEF-binding site within the promoter region of $N U M B$ and $N U M B L$ genes were searched for in this study. Because two TCF/LEF-binding sites were identified within human $N U M B$ promoter based on bioinformatics and human intelligence, comparative integromics analyses on NUMB orthologs were further performed.

Chimpanzee NUBM gene, consisting of 13 exons, was identified within NW_115880.1 genome sequence (Fig. 1). XM_510045.1 was not the correct coding sequence for chimpanzee NUMB. Chimpanzee NUMB gene was found to encode a 651-amino-acid protein showing 99.5, 93.9 and $82.6 \%$ total-amino-acid identity with human NUMB, mouse Numb and chicken numb, respectively.

The human $N U M B$, chimpanzee $N U M B$, mouse $N u m b$ and rat Numb promoters were located within AC005280.3, NW_115880.1, AC133183.3 and NW_047762.2 genome sequences, respectively. Double TCF/LEF-binding sites were located about 1100 and 350 bp up-stream of the transcription 


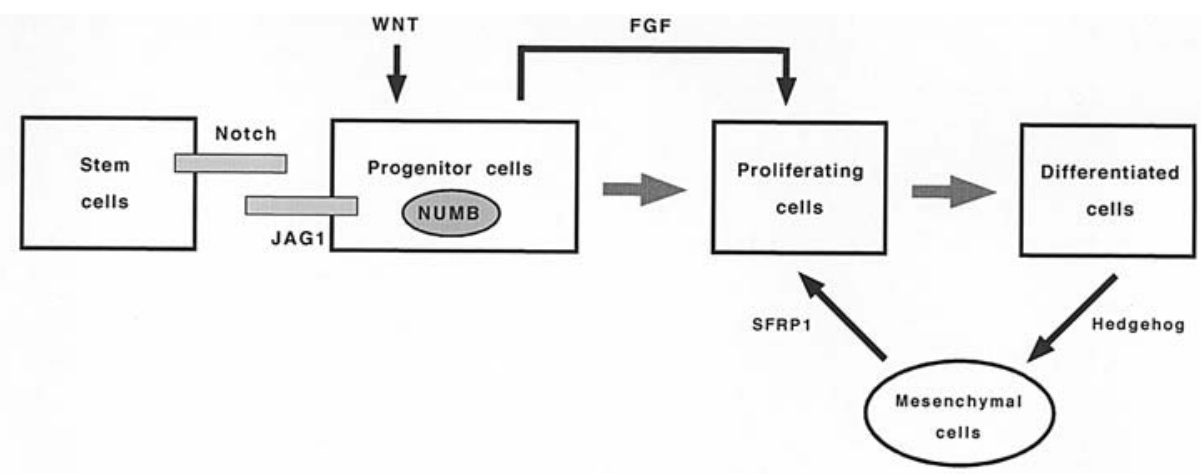

Figure 3. WNT and Notch signaling networks for the homeostasis of stem and progenitor cells. Canonical WNT activates the B-catenin - TCF signaling cascade to induce NUMB and JAG1 expression in progenitor cells. NUMB inhibits Notch signaling in progenitor cells to induce differentiation, while JAG1 activates Notch signaling in stem cells to maintain self-renewal potential.

start site of human $N U M B$ gene. Although distal TCF/LEFbinding site was conserved only among primates, proximal TCF/LEF-binding site was con-served among primates and rodents (Fig. 2). NUMB and JAG1 are potent targets of the canonical WNT signaling pathway in progenitor cells. NUMB inhibits Notch signaling in progenitor cells to induce differentiation, while JAG1 activates Notch signaling in stem cells to maintain self-renewal potential (Fig. 3). Notch signaling inhibitor NUMB was identified as the safe apparatus for the WNT - Notch signaling cycle.

In silico expression analyses in this study revealed that human NUMB mRNA was expressed in placenta, ES cells, neural tissues, trachea, testis, uterus, thymus, coronary artery as well as a variety of tumors, such as cervical cancer, tong tumor, brain tumor, colorectal cancer and breast cancer. NUMBmediated Notch signaling inhibition is lost in a large proportion of human breast cancer due to ubiquitination-dependent NUMB degradation, which results in Notch signaling activation and induction of carcinogenesis $(46,47)$. $N U M B$ is a tumor suppressor gene maintaining homeostasis of stem and progenitor cells through the inhibition of Notch signaling.

Epigenetic changes of cancer-associated genes occur during chronic persistent inflammation, and then genetic alterations of cancer-associated genes occur during multistage carcinogenesis (48-51). Because epigenetic change and genetic alteration of $N U M B$ gene could lead to carcinogenesis through the dysregulation of the WNT - Notch signaling cycle, epigenetic silencing, deletion and loss-offunction mutation of $N U M B$ gene in a variety of human tumors should be investigated in the future.

\section{References}

1. Artavanis-Tsakonas S, Rand MD and Lake RJ: Notch signaling: cell fate control and signal integration in development. Science 284: 770-776, 1999.

2. Radtke F and Raj K: The role of Notch in tumorigenesis: oncogene or tumor suppressor? Nat Rev Cancer 3: 765-767, 2003.

3. Katoh $\mathrm{M}$ and Katoh $\mathrm{M}$ : Identification and characterization of human HES2, HES3, and HES5 genes in silico. Int J Oncol 25: 529-534, 2004.

4. Katoh $\mathrm{M}$ and Katoh M: Identification and characterization of human HESL, rat Hesl and rainbow trout hesl genes in silico. Int J Mol Med 14: 747-751, 2005.

5. Li JL and Harris AL: Notch signaling from tumor cells: a new mechanism of angiogenesis. Cancer Cell 8: 1-3, 2005.
6. Katoh Y and Katoh M: Comparative genomics on HHIP family orthologs. Int J Mol Med 17: 391-395, 2006.

7. Katoh M and Katoh M: Notch ligand, JAG1, is evolutionarily conserved target of canonical WNT signaling pathway in progenitor cells. Int J Mol Med 17: 681-685, 2006.

8. Katoh M and Katoh M: Comparative genomics on FGF8, FGF17, and FGF18 orthologs. Int J Mol Med 16: 493-496, 2005.

9. Katoh $\mathrm{M}$ and Katoh M: Comparative genomics on FGF20 orthologs. Oncol Rep 14: 287-290, 2005.

10. Robbins J, Blondel BJ, Gallahan D and Callahan R: Mouse mammary tumor gene int-3: a member of the Notch gene family transforms mammary epithelial cells. J Virol 66: 2594-2599, 1992.

11. Katoh M: $W N T$ and $F G F$ gene clusters. Int J Oncol 21: 1269-1273, 2002.

12. Katoh M, Hirai M, Sugimura T and Terada M: Cloning, expression and chromosomal localization of WNT13, a novel member of the WNT gene family. Oncogene 13: 873-876, 1996.

13. Saitoh T and Katoh M: Molecular cloning and characterization of human WNTSA. Int J Oncol 19: 123-127, 2001.

14. Katoh M: Frequent up-regulation of WNT2 in primary gastric cancer and colorectal cancer. Int J Oncol 19: 1003-1007, 2001.

15. Kirikoshi H, Sekihara H and Katoh M: WNT10A and WNT6, clustered in human chromosome 2q35 region with head to tail manner, are strongly co-expressed in SW480 cells. Biochem Biophys Res Commun 283: 798-805, 2001.

16. Saitoh T, Hirai M and Katoh M: Molecular cloning and characterization of $W N T 3 A$ and $W N T 14$ clustered in human chromosome 1q42 region. Biochem Biophys Res Commun 284: 1168-1175, 2001

17. Saitoh T, Mine T and Katoh M: Up-regulation of WNT8B mRNA in human gastric cancer. Int J Oncol 20: 343-348, 2002.

18. Heller RS, Klein T, Ling Z, Heimberg H, Katoh M, Madsen OD and Serup P: Expression of WNT, Frizzled, $s F R P$, and $D K K$ genes in adult human pancreas. Gene Expr 11: 141-147, 2003.

19. Katoh $M$ and Katoh M: Identification and characterization of human $B C L 9 L$ gene and mouse Bcl9l gene in silico. Int J Mol Med 12: 643-649, 2003

20. Garciadiego-Cazares D, Rosales C, Katoh M and ChimalMonroy J: Coordination of chondrocyte differentiation and joint formation by $\alpha 5 \beta 1$ integrin in the developing appendicular skeleton. Development 131: 4735-4742, 2004.

21. Swain RK, Katoh M, Medina A and Steinbeisser H: Xenopus frizzled-4S, a splicing variant of Xfz4, is a context-dependent activator and inhibitor of Wnt/B-catenin signaling. Cell Commun Signal 3: 12, 2005.

22. Katoh M: WNT2B: comparative integromics and clinical application. Int J Mol Med 16: 1103-1108, 2005.

23. Katoh M: Epithelial-mesenchymal transition in gastric cancer. Int J Oncol 27: 1677-1683, 2005.

24. Katoh $\mathrm{M}$ and Katoh M: FGFR2 and WDR11 are neighboring oncogene and tumor suppressor gene on human chromosome 10q26. Int J Oncol 22: 1155-1159, 2003.

25. Katoh $\mathrm{M}$ and Katoh M: Evolutionary conservation of CCND1ORAOV1-FGF19-FGF4 locus from zebrafish to human. Int $\mathrm{J}$ Mol Med 12: 45-50, 2003.

26. Katoh $\mathrm{M}$ and Katoh M: Comparative genomics on mammalian FGF3 - FGF4 locus. Int J Oncol 27: 281-285, 2005. 
27. Katoh Y and Katoh M: Comparative genomics on FGF11 orthologs. Oncol Rep 14: 291-294, 2005.

28. Katoh Y and Katoh M: Comparative genomics on mammalian Fgf6 - Fgf23 locus. Int J Mol Med 16: 355-358, 2005.

29. Katoh $\mathrm{Y}$ and Katoh M: Comparative genomics on $F G F 7$, $F G F 10, F G F 22$ orthologs, and identification of $f g f 25$. Int J Mol Med 16: 767-770, 2005.

30. Katoh Y and Katoh M: Comparative genomics on FGF16 orthologs. Int J Mol Med 16: 959-963, 2005.

31. Katoh Y and Katoh M: FGF signaling inhibitor, SPRY4, is evolutionarily conserved target of WNT signaling pathway in progenitor cells. Int J Mol Med 17: 529-532, 2006.

32. Sherrington R, Rogaev EI, Liang Y, et al: Cloning of a gene bearing missense mutations in early-onset familial Alzheimer's disease. Nature 375: 754-760, 1995.

33. Wong WT, Schumacher C, Salcini AE, et al: A protein-binding domain, EH, identified in the receptor tyrosine kinase substrate Eps15 and conserved in evolution. Proc Natl Acad Sci USA 92: 9530-9534, 1995

34. Katoh M: Paradigm shift in gene-finding method: from benchtop approach to desk-top approach. Int J Mol Med 10: 677-682, 2002.

35. Katoh $\mathrm{Y}$ and Katoh M: Identification and characterization of rat Wnt6 and Wnt10a genes in silico. Int J Mol Med 15: 527-531, 2005.

36. Katoh M and Katoh M: Identification and characterization of rat Ror1 and Ror2 genes in silico. Int J Mol Med 15: 533-538, 2005.

37. Katoh Y and Katoh M: Comparative genomics on DKK1 orthologs. Int J Oncol 27: 275-279, 2005.

38. Katoh Y and Katoh M: Comparative genomics on $D K K 2$ and DKK4 orthologs. Int J Mol Med 16: 477-481, 2005.
39. Katoh Y and Katoh M: Comparative genomics on SLIT1, SLIT2, and SLIT3 orthologs. Oncol Rep 14: 1351-1355, 2005.

40. Katoh $\mathrm{Y}$ and Katoh M: Identification and characterization of rat Wnt1 and Wnt10b genes in silico. Int J Oncol 26: 841-845, 2005.

41. Katoh M and Katoh M: Comparative genomics on WNT8A and WNT8B genes. Int J Oncol 26: 1129-1133, 2005.

42. Katoh M: Molecular evolution of WNT2B orthologs. Int J Oncol 26: 1135-1139, 2005.

43. Katoh M: Comparative genomics on WNT3-WNT9B gene cluster. Int J Mol Med 15: 743-747, 2005.

44. Katoh $\mathrm{M}$ and Katoh M: Comparative genomics on WNT5A and WNT5B genes. Int J Mol Med 15: 749-753, 2005.

45. Katoh Y and Katoh M: Comparative genomics on WNT11 gene. Int J Mol Med 15: 879-883, 2005.

46. Pece S, Serresi M, Santolini E, et al: Loss of negative regulation by Numb over Notch is relevant to human breast carcinogenesis. J Cell Biol 167: 215-221, 2004.

47. Stylianou S, Clarke RB and Brennan K: Aberrant activation of notch signaling in human breast cancer. Cancer Res 66: 1517-1525, 2006.

48. Katoh M, Katoh M: Pharmacogenomics on gastric cancer. Cancer Biol Ther 3: 566-567, 2004.

49. Katoh $\mathrm{Y}$ and Katoh M: Hedgehog signaling in gastric cancer. Cancer Biol Ther 4: 1050-1054, 2005.

50. Katoh M: Bioinformatics for cancer management in the postgenome era. Technol Cancer Res Treat 5: 169-176, 2006.

51. Baylin SB and Ohm JE: Epigenetic gene silencing in cancer - a mechanism for early oncogenic pathway addiction? Nat Rev Cancer 6: 107-116, 2006. 